\title{
Disponibilidade e mineralização do nitrogênio após aplicações sucessivas de lodo de esgoto no solo, estimadas por meio de incubação anaeróbica
}

\author{
Adriana Marlene Moreno Pires ${ }^{(1)}$, Cristiano Alberto de Andrade(1), Nadiane Aparecida Pereira de Souza(2), \\ Janaina Braga do Carmo ${ }^{(3)}$, Aline Renee Coscione $^{(2)}$ e Cristina Silva Carvalho ${ }^{(2)}$
}

\begin{abstract}
(1)Embrapa Meio Ambiente, Rodovia SP-340, Km 127, Caixa Postal 69, CEP 13820-000 Jaguariúna, SP, Brasil. E-mail: adriana.pires@embrapa.br, cristiano.andrade@embrapa.br (2)Instituto Agronômico, Avenida Barão de Itapura, oㅡ 1.481, Botafogo, CEP 13012-970 Campinas, SP, Brasil. E-mail: nadisouza@yahoo.com.br, aline@iac.sp.gov.br, criscarvalho25@yahoo.com.br (3)Universidade Federal de São Carlos, Campus Sorocaba, Rodovia SP-264, Km 110, Itinga, CEP 18052-780 Sorocaba, SP, Brasil. E-mail: jbcarmo2008@gmail.com
\end{abstract}

Resumo - O objetivo deste trabalho foi avaliar, por meio de incubação anaeróbica, a mineralização e a disponibilidade de $\mathrm{N}$ em solos tratados com aplicações sucessivas de lodo de esgoto, e determinar o efeito residual das aplicações anteriores na taxa de mineralização do nitrogênio (TMN). Dois experimentos de longa duração foram realizados com doses anuais de lodo de esgoto, para o cultivo de milho: um com dose recomendada de lodo, correspondente a $120 \mathrm{~kg} \mathrm{ha}^{-1}$ de $\mathrm{N}$, e 2, 4 e 8 vezes essa dose, em área da Embrapa Meio Ambiente, em Jaguariúna; e outro com $80 \mathrm{~kg} \mathrm{ha}^{-1}$ de $\mathrm{N}$ aplicado via adubo mineral, e 1 e 2 vezes a dose recomendada de lodo de esgoto, em área do Instituto Agronômico, em Campinas; além de testemunha, sem aplicação de lodo. A disponibilidade de $\mathrm{N}$ e a TMN foram estimadas em condições de campo e por incubações anaeróbicas em laboratório. $\mathrm{O} \mathrm{N}$ total absorvido pelas plantas também foi determinado. O lodo de esgoto é mais efetivo em fornecer nitrogênio ao milho, em longo prazo, do que a adubação mineral. $\mathrm{O}$ efeito residual do lodo de esgoto não influencia a TMN do lodo recém-adicionado. O método de incubação anaeróbica é eficiente em estimar a mineralização do $\mathrm{N}$ proveniente de lodo de esgoto recém-adicionado.

Termos para indexação: Zea mays, acúmulo de nitrato, efeito residual, fertilização orgânica, resíduo orgânico.

\section{Nitrogen availability and mineralization after successive sewage sludge applications on soil, estimated through anaerobic incubation}

\begin{abstract}
The objective of this work was to evaluate, through anaerobic incubation, $\mathrm{N}$ mineralization and availability in soils treated with successive applications of sewage sludge, and to determine the residual effect of previous applications on nitrogen mineralization rate (NMR). Two long-term experiments were conducted with annual sewage sludge doses for corn cultivation: one with the recommended dose of sewage sludge, correspondent to $120 \mathrm{~kg} \mathrm{ha}^{-1} \mathrm{~N}$, and 2, 4, and 8 times the dose in area at Embrapa Meio Ambiente, in Jaguariúna, in the state of São Paulo, Brazil; and the other with $80 \mathrm{~kg} \mathrm{ha}^{-1}$ mineral $\mathrm{N}$ fertilization, and 1 and 2 times the recommended dose of sewage sludge in area at Instituto Agronômico, in Campinas, in the state of São Paulo, Brazil; besides a control, without sewage sludge application. $\mathrm{N}$ availability and NMR were determined in field conditions and through anaerobic incubations in laboratory. Total nitrogen absorbed by plants was also estimated. Sewage sludge is more effective in supplying nitrogen to corn in the long term than mineral fertilization. The residual effect of sewage sludge does not influence the NMR from newly-added sludge. The method of anaerobic incubation is efficient in estimating the mineralization of $\mathrm{N}$ from newly-added sewage sludge.
\end{abstract}

Index terms: Zea mays, nitrate accumulation, residual effect, organic fertilization, organic waste.

\section{Introdução}

O uso agrícola do lodo de esgoto no Brasil é regulamentado pela Resolução Conama $\mathrm{n}^{\mathrm{o}} 375$ (Brasil, 2006), na qual estão indicados os critérios e os procedimentos para seu uso adequado. Entre esses procedimentos, recomenda-se que o cálculo da dose de aplicação do resíduo baseie-se em três parâmetros: elevação de pH, acúmulo de metais pesados no solo e quantidade nitrogênio disponível. Deve-se utilizar a menor dose calculada a partir desses parâmetros, que geralmente é a obtida com base na disponibilidade de N. 
Neste caso, a dose $\left(\mathrm{Mg} \mathrm{ha}^{-1}\right)$ é estimada pelo quociente entre a quantidade de nitrogênio $\left(\mathrm{kg} \mathrm{ha}^{-1}\right)$ indicada para a cultura, segundo o Boletim 100 (Van Raij et al., 1997), e a quantidade de nitrogênio disponível no lodo de esgoto $\left(\mathrm{kg} \mathrm{Mg}^{-1}\right)$.

$\mathrm{O}$ nitrogênio presente no resíduo encontra-se predominantemente na forma orgânica. Assim, sua disponibilidade a partir do lodo depende da mineralização do resíduo (Andrade et al., 2010), que envolve processos essencialmente microbiológicos, dependentes das características do próprio resíduo, dos atributos do solo e de fatores climáticos (Cantarella et al., 2008).

A quantidade de nitrogênio mineralizável durante o ciclo da cultura varia de acordo com o teor total de $\mathrm{N}$ no resíduo, com seu teor de $\mathrm{N}$ inorgânico e com a taxa de mineralização de N (TMN) (Brasil, 2006). Esses parâmetros são obtidos por meio de ensaios em laboratório. O método de incubação aeróbica é o mais adequado para esse tipo de estudo. Andrade et al. (2013) avaliaram o efeito de aplicações sucessivas de lodo de esgoto sobre a TMN por meio da incubação aeróbica; porém, esse método não é rotineiramente utilizado por demandar longo período de avaliação (120 dias), ter custo relativamente alto e ser um procedimento de baixa operacionalidade (Cantarella et al., 2008). Portanto, é importante que se avalie a eficiência de métodos de incubação anaeróbicos, de curta duração, para estimar a mineralização. Nesse método, as amostras de solo são incubadas em condições saturadas, por um período de 7 a 14 dias, para determinação do teor de $\mathrm{N}$ amoniacal. Somente é possível determinar essa forma de $\mathrm{N}$, pois a nitrificação não ocorre na ausência de oxigênio (Keeney, 1982). A incubação anaeróbica é mais simples, rápida e menos dispendiosa que os outros métodos de incubação.

Correlações significativas entre os resultados dos métodos aeróbico e anaeróbico têm sido relatadas (Boeira, 2005; Wienhold, 2007). Além disso, correlações significativas entre o $\mathrm{N}$ potencialmente mineralizável e o $\mathrm{N}$ absorvido pelas plantas também foram constatadas em incubações anaeróbicas em solos dos estados do Rio Grande do Sul e de São Paulo (Rhoden, 2006; Yagi, 2009).

Em razão da baixa operacionalidade da incubação aeróbica, a Resolução Conama no 375 (Brasil, 2006) recomenda valores fixos de TMN, conforme o tipo de lodo e o processo pelo qual foi gerado. Teoricamente, a TMN, determinada em incubação ou definida em função do tipo de lodo, é o parâmetro que define a percentagem do $\mathrm{N}$ orgânico que será mineralizado durante o ciclo da cultura. Os valores de TMN descritos na literatura são geralmente inferiores a 50\%: 5 a 38\% (Alcântara et al., 2007); 14 a 43\% (Boeira \& Maximiliano, 2009); 10 a 52\% (Corrêa et al., 2012); e 7 a 16\% (Andrade et al., 2013). Dessa forma, menos da metade do nitrogênio contido no lodo é disponibilizado ao longo do ciclo das culturas. O restante do $\mathrm{N}$ presente no lodo será mineralizado, provavelmente a menores taxas, ao longo dos anos subsequentes à aplicação.

A adoção de uma TMN fixa pode resultar em doses superestimadas em solos com reaplicação do resíduo, pois desconsidera o efeito residual das aplicações anteriores, o que pode levar ao aumento do $\mathrm{N}$ inorgânico $\left(\mathrm{NO}_{3}^{-}\right)$no solo, que é passível de lixiviação e contaminação de águas subterrâneas (Dynia et al., 2006).

Aplicações sucessivas do lodo de esgoto podem aumentar o teor de $\mathrm{N}$ potencialmente mineralizável no solo, o que sugere a necessidade de se considerar o efeito residual dessas aplicações. Boeira \& Maximiliano (2009) relataram que, de fato, há efeito das aplicações anteriores sobre o potencial de mineralização do nitrogênio, e que doses mais altas levam ao acúmulo de nitrato no solo e representam risco de lixiviação. Em outro experimento de longo prazo, Andrade et al. (2010) verificaram que o nitrogênio potencialmente mineralizável aumenta ao longo de aplicações anuais sucessivas de lodo de esgoto e mesmo um ano após a última aplicação do resíduo. Outro ponto importante, ainda pouco abordado, refere-se ao efeito do $\mathrm{N}$ residual do solo na taxa de mineralização do lodo de esgoto recém-adicionado.

O objetivo deste trabalho foi avaliar, por meio de incubação anaeróbica, a mineralização e a disponibilidade de N, em solos tratados com aplicações sucessivas de lodo de esgoto, e determinar o efeito residual das aplicações anteriores na TMN do resíduo recém-adicionado.

\section{Material e Métodos}

A mineralização do nitrogênio foi avaliada em dois experimentos de longa duração, localizados no Instituto Agronômico $\left(22^{\circ} 52^{\prime} \mathrm{S}, 47^{\circ} 04^{\prime} \mathrm{W}\right.$, a $650 \mathrm{~m}$ de altitude), Campinas, SP, e na Embrapa Meio Ambiente 
$\left(22^{\circ} 41^{\prime} \mathrm{S}, 47^{\circ} 00^{\prime} \mathrm{W}\right.$, a $570 \mathrm{~m}$ de altitude), Jaguariúna, SP. No primeiro, foram realizadas sete aplicações anuais de lodo de esgoto, seguidas de cinco anos sem adição do resíduo, totalizando 12 anos após a primeira aplicação (Andrade et al., 2010). No segundo, foram realizadas seis aplicações anuais de lodo de esgoto, seguidas de dois anos sem adição de resíduo. Depois foram realizadas mais quatro adições anuais do resíduo, seguidas de três anos sem aplicação, totalizando 14 anos após a primeira aplicação do lodo de esgoto (Boeira \& Maximiliano, 2009). Os solos das áreas experimentais foram classificados como Latossolo Vermelho eutroférrico (LVe) e Latossolo Vermelho distroférrico ( $\mathrm{LVd})$, respectivamente (Santos et al., 2006). Os experimentos foram instalados e têm sido desenvolvidos de forma independente, pelas diferentes instituições.

No IAC, o experimento foi conduzido em delineamento inteiramente casualizado, com quatro repetições e parcelas experimentais de $100 \mathrm{~m}^{2}$. Entre 2001 e 2007, foram realizadas sete aplicações anuais do lodo de esgoto da Estação de Tratamento de Esgotos (ETE) de Jundiaí, SP. Foram avaliadas as doses de lodo necessárias para o fornecimento de 80 e $160 \mathrm{~kg} \mathrm{ha}^{-1} \mathrm{de} \mathrm{N}$ (tratamentos $1 \mathrm{~N}$ e $2 \mathrm{~N}$, respectivamente) para a cultura do milho (Zea mays L.), bem como a adubação mineral (80 $\left.\mathrm{kg} \mathrm{ha}^{-1}\right)$, calculada para atender a necessidade da cultura. Detalhes deste experimento estão disponíveis em Bueno et al. (2011).

Na Embrapa Meio Ambiente, o lodo de esgoto aplicado era proveniente da ETE de Franca, SP. Foram avaliados os seguintes tratamentos: testemunha, sem adição de lodo $(0 \mathrm{~N})$; dose de lodo necessária para o fornecimento de $120 \mathrm{~kg} \mathrm{ha}^{-1}$ de $\mathrm{N}(1 \mathrm{~N})$, calculada de acordo com a produtividade esperada de $8-10 \mathrm{Mg} \mathrm{ha}^{-1}$ de milho; e 2, 4 e 8 vezes essa dose $(2 \mathrm{~N}, 4 \mathrm{~N}$ e $8 \mathrm{~N}$, respectivamente). Maiores detalhes sobre a instalação e os primeiros anos de condução da área experimental podem ser obtidos em Bettiol et al. (2006).

O lodo de esgoto foi aplicado manualmente e incorporado ao solo na camada de $0-20 \mathrm{~cm}$ de profundidade, na área total. No IAC, a incorporação foi realizada manualmente, e, na Embrapa Meio Ambiente, com enxada rotativa. As adubações minerais foram realizadas em cobertura, ao lado da linha de plantio, como indicado no Boletim 100 (Van Raij et al., 1997). Na maioria dos anos, as fontes utilizadas foram: formulação NPK 04-14-08 no plantio e nitrato de amônio em cobertura. Em todos os tratamentos, foi realizada complementação da adubação potássica, com uso de $\mathrm{KCl}$ em cobertura.

Em outubro de 2012, foram retiradas amostras de solo da camada de 0 a $20 \mathrm{~cm}$, nos dois experimentos. Em dezembro, novas aplicações de lodo de esgoto, proveniente da ETE de Jundiaí, SP (Tabela 1), foram realizadas nas duas áreas experimentais. Para o cálculo da dose recomendada, adotou-se TMN de 30\%, indicada para o tipo de lodo aplicado (Brasil, 2006), e necessidade da cultura igual a $120 \mathrm{~kg} \mathrm{ha}^{-1}$ de $\mathrm{N}$, conforme o Boletim 100 (Van Raij et al., 1997), para a cultura de milho de alta produtividade, destinado à produção de grãos e silagem.

$\mathrm{Na}$ safra 2012/2013, ambos os experimentos receberam adubação mineral, na dose de $30 \mathrm{~kg} \mathrm{ha}^{-1}$ de $\mathrm{N}$ no plantio (formulação 04-14-08) e de $90 \mathrm{~kg} \mathrm{ha}^{-1}$ de $\mathrm{N}$ em cobertura (nitrato de amônio), 30 dias após o plantio. A adubação potássica foi utilizada em todos os tratamentos, com $\mathrm{KCl}\left(30 \mathrm{~kg} \mathrm{ha}^{-1}\right.$ de $\left.\mathrm{K}_{2} \mathrm{O}\right)$. Nas parcelas do tratamento testemunha, na Embrapa Meio Ambiente, não foi realizada adição de nenhum fertilizante nitrogenado.

O plantio do milho (híbrido simples precoce de alto potencial produtivo, $2 \mathrm{~B} 587 \mathrm{Hx}$ ), nos dois experimentos, foi feito com espaçamento de $0,45 \mathrm{~m}$ nas entrelinhas. Por ocasião da colheita, as plantas foram amostradas, secas a $65^{\circ} \mathrm{C}$ e moídas, para determinação da produção de massa seca e do teor total de nitrogênio, determinado por combustão a seco em analisador elementar Leco

Tabela 1. Atributos químicos do lodo de esgoto utilizado na incubação anaeróbica e aplicado nas áreas experimentais da Embrapa Meio Ambiente e do Instituto Agronômico, em 2012.

\begin{tabular}{lc}
\hline Atributo $^{(1)}$ & Valor \\
\hline Umidade a $65^{\circ} \mathrm{C}\left({\left.\mathrm{g} 100 \mathrm{~g}^{-1}\right)}_{\mathrm{pH} \text { em } \mathrm{H}_{2} \mathrm{O}}\right.$ & 63,00 \\
Carbono $\left(\mathrm{g} \mathrm{kg}^{-1}\right)$ & 7,21 \\
Sólidos voláteis $(\%)$ & 315,55 \\
Sólidos totais $(\%)$ & 51,30 \\
Capacidade de troca catiônica & 33,13 \\
$\mathrm{~N}$ Kjeldahl $\left(\mathrm{g} \mathrm{kg}^{-1}\right)$ & 218,48 \\
$\mathrm{~N}$ amoniacal $\left(\mathrm{g} \mathrm{kg}^{-1}\right)$ & 29,39 \\
$\mathrm{~N}$ nítrico $\left(\mathrm{g} \mathrm{kg}^{-1}\right)$ & 0,70 \\
Fósforo $\left(\mathrm{g} \mathrm{kg}^{-1}\right)$ & 0,18 \\
\hline
\end{tabular}

${ }^{(1)}$ Exceto para umidade, as concentrações estão expressas com base na matéria seca.

Pesq. agropec. bras., Brasília, v.50, n.4, p.333-342, abr. 2015 DOI: 10.1590/S0100-204X2015000400009 
CN-2000 (Leco Corporation, St. Joseph, MI, EUA). Os resultados foram submetidos à análise de variância e de regressão, no caso da área experimental da Embrapa Meio Ambiente. No experimento do IAC, utilizou-se o teste de Tukey, para comparação das médias.

A quantidade de nitrogênio mineralizado in situ foi estimada apenas no experimento da Embrapa, por meio da instalação de tubos de PVC $(30 \mathrm{~cm}$ de comprimento e $5 \mathrm{~cm}$ de diâmetro), na camada de 0 a $20 \mathrm{~cm}$ (Adams \& Attiwill, 1986). Em cada parcela, foram instalados três conjuntos de dois tubos, um com a extremidade superior aberta e outro com ela fechada. As diferenças entre as quantidades mineralizadas nos tubos fechados e abertos representaram as quantidades de $\mathrm{N}$ inorgânico percoladas. Em cada instalação dos tubos, aos 20, 50 e 80 dias após a incorporação do lodo de esgoto, o solo foi amostrado para determinação do $\mathrm{N}$ na forma inorgânica. Os tubos permaneceram no solo por períodos pré-determinados (20 a 50,50 a 80 e 80 a 110 dias após a incorporação do lodo de esgoto). Ao final de cada período, o conjunto de tubos foi retirado e um novo conjunto foi instalado.

$\mathrm{O}$ cálculo da quantidade de $\mathrm{N}$ mineralizado $\left(\mathrm{N}_{\min }\right)$ em cada período foi realizado da seguinte forma: $\mathrm{N}_{\min }$ total $=\mathrm{N}_{\min }$ na retirada dos tubos $-\mathrm{N}_{\min }$ na instalação dos tubos. A quantidade total de $\mathrm{N}$ mineralizado aos 110 dias foi dada pela soma das quantidades mineralizadas em cada período de avaliação. As amostras coletadas foram homogeneizadas no campo e acondicionadas sob refrigeração para posterior análise dos teores de $\mathrm{N}$ inorgânico (soma de nitrato e amônio). A extração foi realizada com solução

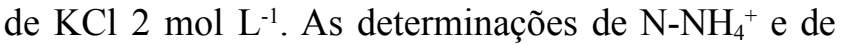
$\mathrm{N}-\mathrm{NO}_{3}$ foram feitas em extratos, em sistema automático de injeção em fluxo contínuo (FIA) (Krom, 1980) e com leitura colorimétrica (Kamphake et al., 1967).

Os dados foram submetidos à análise de variância, tendo-se utilizado o teste de Tukey para comparação das médias dos tipos de tubo, e a análise de regressão para avaliação dos efeitos das doses de lodo ao longo do tempo.

$\mathrm{Na}$ avaliação da TMN em condições controladas, foram retiradas amostras de solo da camada de $0-20 \mathrm{~cm}$, antes da aplicação do lodo. As amostras foram secas ao ar e peneiradas em malha de $2 \mathrm{~mm}$ para, então, receberem novas doses de lodo de esgoto (Tabela 1 ): $60,120,240$ e $480 \mathrm{~kg} \mathrm{ha}^{-1}$ de $\mathrm{N}$ disponível, tendo-se considerado a dose de $120 \mathrm{~kg} \mathrm{ha}^{-1}$ como a recomendada para o cultivo do milho de alta produtividade; além de testemunha, sem adição de lodo. Portanto, avaliaramse dois fatores: as doses recém-adicionadas e o efeito residual, que se refere ao histórico da área (tratamentos no campo).

A incubação anaeróbica foi realizada de acordo com Keeney (1982), com uso de 5 g de solo de cada tratamento (Embrapa Meio Ambiente: 0N, 1N, $2 \mathrm{~N}, 4 \mathrm{~N}$ e $8 \mathrm{~N}$; e IAC: adubação mineral, $1 \mathrm{~N}$ e $2 \mathrm{~N}$ ), acondicionados em tubos tipo Falcon e acrescidos das novas doses. Os teores de nitrogênio na forma inorgânica foram determinados antes (tempo inicial) e após 7 dias (tempo final) de incubação. Para cada conjunto dose/ tratamento/tempo, foram montados quatro tubos, que corresponderam às repetições.

Para a determinação dos teores iniciais de nitrogênio inorgânico, foram adicionados $25 \mathrm{~mL}$ de $\mathrm{KCl} 2 \mathrm{~mol} \mathrm{~L}^{-1}$ aos tubos, que foram agitados a $120 \mathrm{rpm}$, por $30 \mathrm{~min}$. Para a determinação dos teores finais, adicionaram-se 12,5 mL de água deionizada a cada tubo, para garantir a condição de saturação; em seguida, os tubos foram acondicionados em estufas incubadoras tipo BOD, com temperatura de $40^{\circ} \mathrm{C} \pm 1$, por 7 dias. Ao final deste período, foram acrescentados $12,5 \mathrm{~mL}$ de solução de $\mathrm{KCl} 4 \mathrm{~mol} \mathrm{~L}^{-1}$, tendo-se agitado os tubos a $120 \mathrm{rpm}$, durante $30 \mathrm{~min}$. Os conteúdos dos tubos foram filtrados para obtenção dos extratos.

Os fatores de variação (efeito residual e as novas doses de lodo) foram avaliados em arranjos fatoriais $5 \times 5$ e $3 \times 5$, respectivamente para as áreas da Embrapa Meio Ambiente e do IAC. Os dados foram submetidos à análise de variância e de regressão (novas doses), e as médias para efeito residual foram comparadas pelo teste de Tukey (IAC) ou foram submetidas a análise de regressão (Embrapa). Realizou-se, também, a análise de correlação entre os dados obtidos em laboratório e no campo, para avaliar a capacidade do método anaeróbico em estimar o $\mathrm{N}$ mineralizado.

\section{Resultados e Discussão}

As doses de lodo de esgoto adicionadas ao solo influenciaram as quantidades totais de $\mathrm{N}$ absorvido pelas plantas de milho, em ambas as áreas experimentais (Figura 1). No IAC, a quantidade de nitrogênio absorvido foi $146 \mathrm{~kg} \mathrm{ha}^{-1}$ maior no tratamento $1 \mathrm{~N}$ do que com adubação mineral; ou seja, o solo que recebeu a dose recomendada de $\mathrm{N}$ via lodo de esgoto disponibilizou o dobro do nutriente para as plantas, em 
comparação à adubação mineral. Esta grande diferença provavelmente está relacionada ao efeito residual do lodo aplicado anteriormente. Pitombo (2011) relatou que o estoque de $\mathrm{N}$ no solo da área experimental do IAC (0-20 cm), em 2009, aumentou 33\% entre os tratamentos com adubação mineral e $1 \mathrm{~N}$, tendo passado de 2,6 para 3,5 $\mathrm{Mg} \mathrm{ha}^{-1}$ de N. Portanto, as sucessivas aplicações de lodo de esgoto incrementam significativamente a disponibilidade de $\mathrm{N}$ no solo, quer seja pelo aumento do estoque do nutriente no solo, como demonstrado por Pitombo (2011), quer seja pela
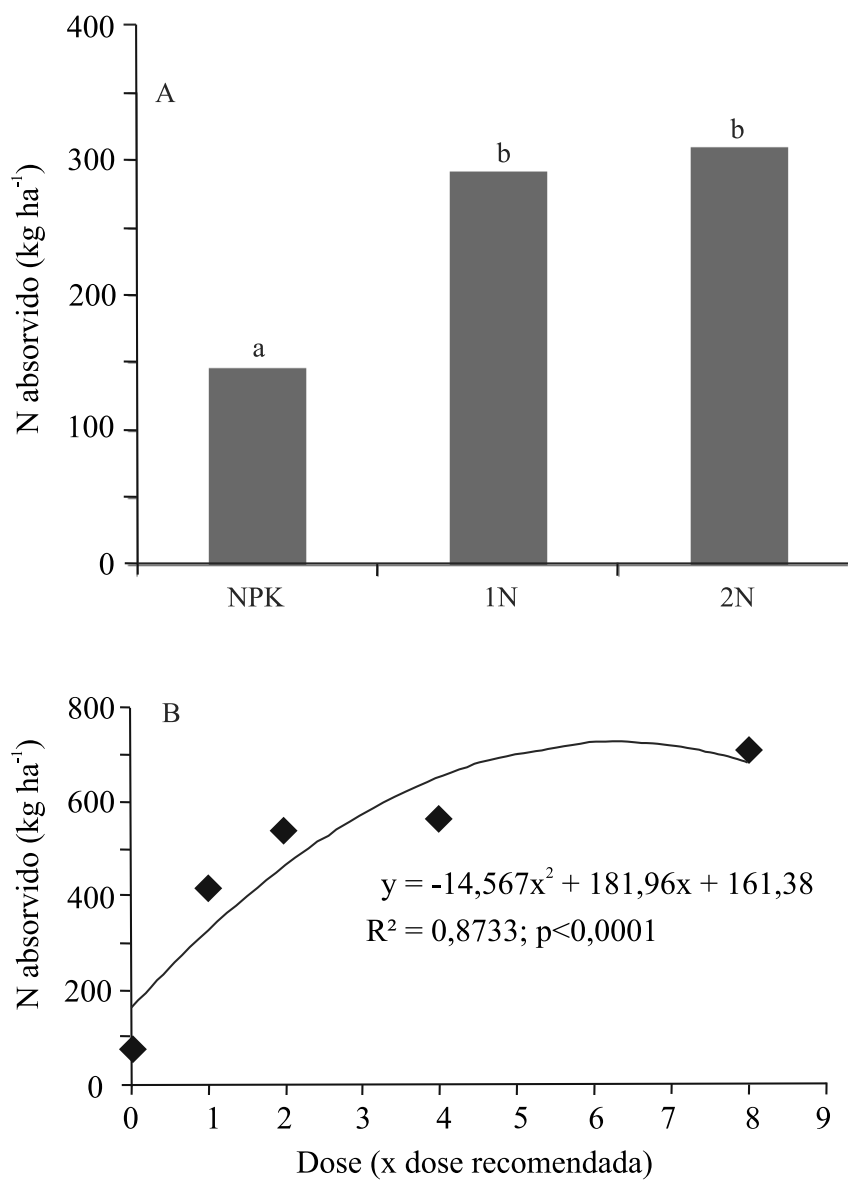

Figura 1. Nitrogênio absorvido por plantas de milho (Zea mays) em função da aplicação de doses de lodo de esgoto nos experimentos realizados no Instituto Agronômico (A) e na Embrapa Meio Ambiente (B). Em A, médias seguidas de letras iguais não diferem pelo teste de Tukey, a $10 \%$ de probabilidade. $0 \mathrm{~N}$, sem aplicação de lodo; $1 \mathrm{~N}$, dose de lodo de esgoto recomendada, correspondente a $120 \mathrm{~kg} \mathrm{ha}^{-1} \mathrm{de} \mathrm{N}$, ao se considerar taxa de mineralização de $\mathrm{N}$ igual a $30 \%$ (Brasil, 2006); e 2N, $4 \mathrm{~N}$ e $8 \mathrm{~N}$, doses correspondentes a 2, 4 e 8 vezes a dose recomendada. alteração da TMN do solo ou do lodo recém-aplicado. Dessa forma, o sistema de produção com lodo de esgoto proporciona maior eficiência no fornecimento de $\mathrm{N}$ em longo prazo.

No experimento realizado na Embrapa Meio Ambiente, a relação entre a quantidade de $\mathrm{N}$ absorvido pelas plantas e a dose de lodo aplicada foi ajustada por meio de um modelo quadrático (Figura 1). Esse resultado era esperado, uma vez que as plantas comumente respondem ao aumento na dose de insumos com incrementos decrescentes, até o ponto em que outro fator limitante ao crescimento passe a predominar (Taiz \& Zeiger, 1998). Em razão da limitada capacidade de absorção de $\mathrm{N}$ pelas plantas, aplicações excessivas do nutriente favorecem perdas por lixiviação. No presente trabalho, a dose que correspondeu à maior absorção de $\mathrm{N}$ pelas plantas foi a equivalente a 6,3 vezes a dose recomendada de $120 \mathrm{~kg} \mathrm{ha}^{-1}$ de $\mathrm{N}$. Doses maiores que essa não aumentaram a absorção de nitrogênio.

Não foi possível estimar a mineralização de $\mathrm{N}$ no campo, uma vez que os valores obtidos nos tempos iniciais dos períodos avaliados não foram diferentes dos verificados após a permanência no campo, independentemente do tipo de tubo instalado (Tabela 2). Essa semelhança não era esperada, pois havia expectativa de maiores teores ao final do período e com o uso de tubos fechados, já que os tubos abertos possibilitam perdas por lixiviação. No entanto, a extrema variabilidade observada nos valores de $\mathrm{N}$ mineralizado (CV de 147\%) impossibilitou a identificação de diferença entre os tratamentos, o que corrobora a noção de que os valores de $\mathrm{N}$ inorgânico $\left(\mathrm{N}-\mathrm{NH}_{4}+\mathrm{N}-\mathrm{NO}^{3-}\right)$ são indicadores pobres da disponibilidade do nutriente no solo em condições não controladas (Stevenson, 1986).

Tabela 2. Média dos teores de $\mathrm{N}$ inorgânico no momento da instalação dos conjuntos de tubos (abertos ou fechados) no campo, aos 20, 50 e 80 dias após a incorporação do lodo e após $30 \operatorname{dias}^{(1)}$.

\begin{tabular}{lc}
\hline Tempo & N inorgânico $\left(\mathrm{mg} \mathrm{kg}^{-1}\right)$ \\
\hline Instalação & $198 \mathrm{a}$ \\
Tubo aberto & $118 \mathrm{a}$ \\
Tubo fechado & $207 \mathrm{a}$ \\
\hline $\mathrm{CV}(\%)$ & 147 \\
\hline${ }^{(1)}$ Médias seguidas de letras iguais não diferem pelo teste de Tukey, a $10 \%$ \\
de probabilidade.
\end{tabular}

Pesq. agropec. bras., Brasília, v.50, n.4, p.333-342, abr. 2015 DOI: 10.1590/S0100-204X2015000400009 
A literatura pertinente é pouco conclusiva quanto aos resultados de mineralização do N (Vieira \& Cardoso, 2003; Gonçalves, 2005). Nesses estudos, os tubos apresentavam furos laterais para, presumivelmente, permitir equilíbrio da umidade do solo entre o ambiente interno do tubo e o externo. No presente trabalho, optou-se por não se fazer os furos, pois eles poderiam representar uma fonte de erro, pela difusão de formas inorgânicas de $\mathrm{N}$ entre os ambientes interno e externo. Como o procedimento com os tubos não permitiu avaliar a mineralização do $\mathrm{N}$, optou-se por estimar a TMN com base nas quantidades de $\mathrm{N}$ na forma inorgânica, em cada instalação dos tubos; ou seja, nas amostragens realizadas aos 20,50 e 80 dias da incorporação do lodo.

Os teores de $\mathrm{N}$ inorgânico no solo variaram de acordo com o tempo de amostragem e com as doses de lodo aplicadas, sem que tenha havido interação significativa entre esses fatores. $\mathrm{O} \mathrm{N}$ inorgânico
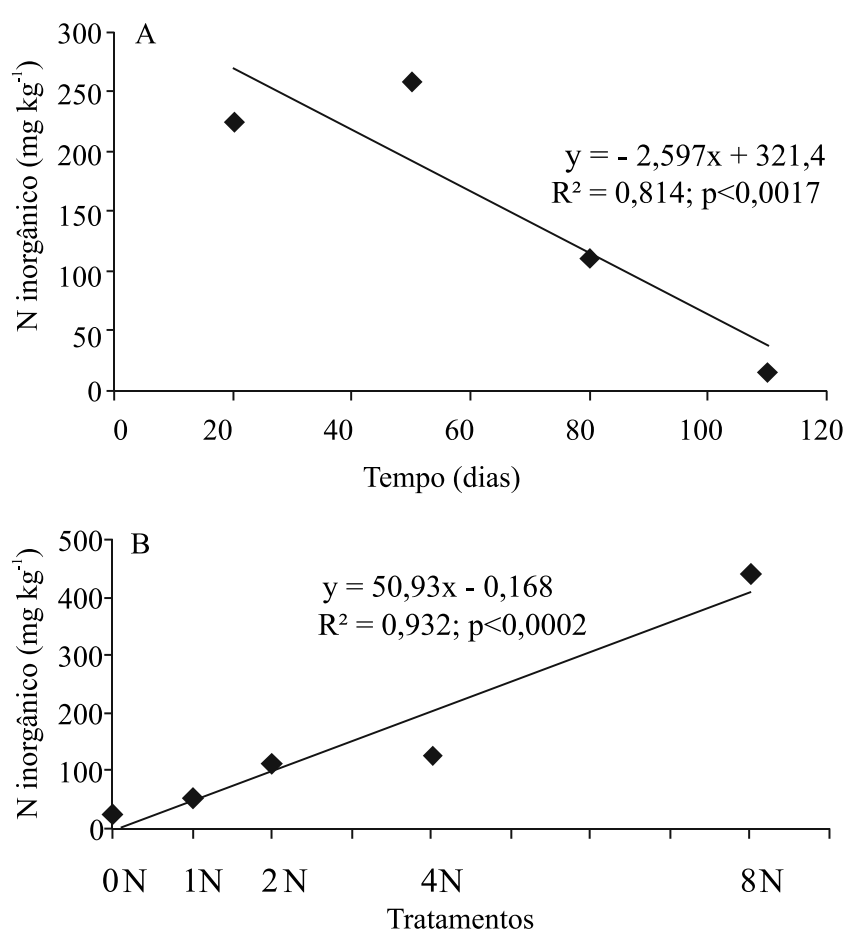

Figura 2. Teor de $\mathrm{N}$ inorgânico no solo em função do tempo após incorporação das doses de lodo de esgoto (A) e em função das doses (B). 0N, sem aplicação de lodo; $1 \mathrm{~N}$, dose de lodo de esgoto recomendada, correspondente a $120 \mathrm{~kg} \mathrm{ha}^{-1}$ de $\mathrm{N}$, ao se considerar taxa de mineralização de $\mathrm{N}$ igual a $30 \%$ (Brasil, 2006); e 2N, $4 \mathrm{~N}$ e $8 \mathrm{~N}$, doses correspondentes a 2,4 e 8 vezes a dose recomendada. diminuiu com o tempo (Figura 2), possivelmente em razão da absorção do nutriente pelas plantas de milho e de perdas, principalmente por lixiviação. Segundo Cantarella \& Duarte (2004), cerca de 50 a $75 \%$ da absorção do $\mathrm{N}$ total acumulado pelo milho ocorre até o florescimento, que acontece por volta dos 60 dias após a emergência. Durante esse período, esperase maior disponibilização de $\mathrm{N}$ pela mineralização do lodo. Conforme Andrade et al. (2013), em estudo com condições controladas de umidade e temperatura, metade do total de $\mathrm{N}$ mineralizado do lodo de esgoto, em 126 dias de avaliação, ocorre entre os primeiros 10 e 14 dias. Isso significa que, com o passar do tempo, a mineralização do $\mathrm{N}$ do lodo ocorre a menores taxas, mas a absorção do $\mathrm{N}$ pela cultura continua, o que leva à redução do teor de $\mathrm{N}$ inorgânico no solo. Vieira \& Cardoso (2003) também observaram redução no teor de nitrato a partir dos 66 dias após a emergência de plantas de milho, em trabalho com lodo de esgoto.

$\mathrm{O}$ teor de N inorgânico no solo aumentou linearmente com o aumento das doses de lodo (Figura 2). Ao se considerarem o teor total de $\mathrm{N}$ no lodo, a quantidade de resíduo adicionada e a quantidade de nitrogênio disponibilizada pelo resíduo nas diferentes doses, foram disponibilizados $7,0 \mathrm{~kg}$ de $\mathrm{N}$ por tonelada de lodo aplicada (coeficiente angular da equação de regressão) em 1 ha (profundidade de incorporação igual a $20 \mathrm{~cm}$ e densidade do solo igual a $1,0 \mathrm{Mg} \mathrm{m}^{-3}$ ). A partir desse coeficiente angular $\left(7,0 \mathrm{~kg} \mathrm{Mg}^{-1}\right)$ e do $\mathrm{N}$ total aplicado $\left(29,4 \mathrm{~kg} \mathrm{Mg}^{-1}\right)$, estimou-se a TMN em $24 \%$. Essa taxa não chegou aos 30\% indicados na Resolução no 375 (Brasil, 2006). Vale ressaltar, no entanto, que, neste caso, não foram consideradas as prováveis perdas por lixiviação nem as quantidades absorvidas pelo milho.

Dessa forma, para melhorar a estimativa do $\mathrm{N}$ mineralizado em campo, procedeu-se à linearização da equação de regressão entre as doses de $\mathrm{N}$ e a quantidade absorvida do nutriente pela cultura (Figura 1). Por meio desse artifício, chegou-se a equação: $\mathrm{y}=29,12 \mathrm{x}+181,9$, o que indica que $29,12 \mathrm{~kg} \mathrm{ha}^{-1} \mathrm{de}$ $\mathrm{N}$ são absorvidos pelo milho para cada $14,6 \mathrm{Mg} \mathrm{ha}^{-1}$ de lodo aplicado. Assim, para cada tonelada de lodo aplicada, 2,0 kg de $\mathrm{N}$ foram absorvidos pela cultura. Portanto, a soma deste valor aos 7,0 kg de $\mathrm{N}$ disponível no solo, por tonelada de lodo aplicado, representaria uma TMN de $31 \%$.

$\mathrm{Na}$ avaliação dos efeitos residual e de novas doses de lodo aplicadas, apenas o fator novas doses foi 
significativo para a quantidade de $\mathrm{N}$ mineralizado. $\mathrm{O}$ teor de $\mathrm{N}$ mineralizado no solo aumentou linearmente com as novas doses de lodo, o que era esperado (Figura 3). Boeira et al. (2002) e Alcântara et al. (2007) também observaram incrementos de $\mathrm{N}$ proporcionais às doses de lodo aplicadas. Andrade et al. (2013), ao estudar doses de resíduos em área com histórico de uso anterior de lodo de esgoto, também encontraram aumento na concentração de $\mathrm{N}$ com as doses aplicadas, embora, nesse caso, tenha havido, adicionalmente, efeito de aplicações anteriores do resíduo.

ONmineralizado, oriundo da dose recém-adicionada, é estimado pela subtração dos valores de $\mathrm{N}$ inorgânico, presentes no tempo inicial e na dose zero, dos valores presentes nos diferentes momentos de análise, nas diversas doses aplicadas. Desse modo, o efeito residual de aplicações anteriores mistura-se ao efeito das novas aplicações e pode alterar a taxa de mineralização da nova dose. Isso poderia explicar, em parte, a ausência de significância do efeito residual das aplicações anuais anteriores de lodo no solo. Além disso, a nova aplicação poderia ter estimulado a atividade microbiana e aumentado a mineralização de $\mathrm{N}$, em comparação à dose zero, o que é comumente referido como efeito priming (Kuzyakov, 2010). Contudo,

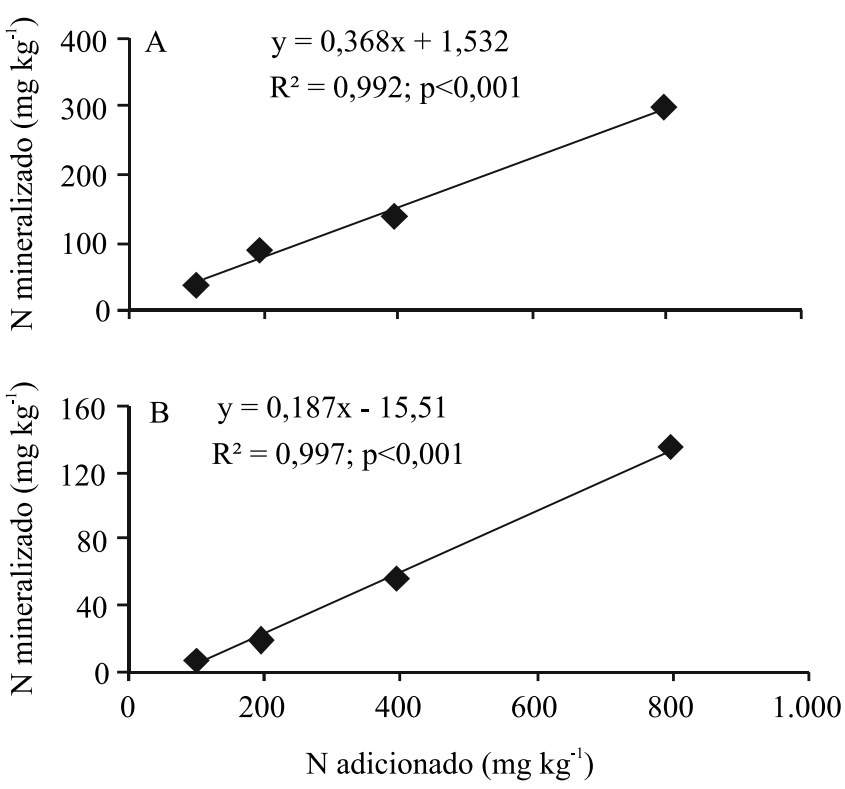

Figura 3. Quantidade de $\mathrm{N}$ mineralizado no solo em função das doses de nitrogênio total recém-adicionadas via lodo de esgoto: A, área experimental do Instituto Agronômico; B, área experimental da Embrapa Meio Ambiente. isso não ocorreu no presente trabalho. Apesar disso, o efeito residual do lodo de esgoto tem sido relatado em vários trabalhos com reaplicação do resíduo (Dynia et al., 2006; Barbosa et al., 2007; Andrade et al., 2013).

Para excluir o efeito da dose e obter um valor de uso mais amplo, que oriente a recomendação da fertilização com o lodo de esgoto, pode-se utilizar os coeficientes angulares das equações presentes na Figura 3 como a TMN do lodo aplicado. Na área experimental do IAC, a TMN foi igual a $37 \%$; porém, na Embrapa Meio Ambiente, ela se reduziu para, praticamente, a metade desse valor: $19 \%$. Como as condições de incubação foram controladas, somente diferenças entre os solos explicariam essa divergência entre as taxas. Assim, a adoção de um valor de TMN apenas em função do tipo de lodo pode não ser a melhor alternativa, e é importante que se preconize a determinação específica, em laboratório, dos valores de TMN para o solo em que se pretende aplicar o resíduo.

Andrade et al. (2013), em trabalho semelhante a este, mas com o método de incubação aeróbico, constataram, na área experimental do IAC, valor médio de TMN de $12 \%$, bem inferior ao obtido no presente trabalho com a incubação anaeróbica (37\%). Boeira \& Maximiliano (2009) obtiveram valores de TMN de $14 \%$, para a incubação anaeróbica, e de $29 \%$ para a aeróbica. Essa variedade de resultados pode ser explicada pelos diferentes resíduos avaliados (Cabrera et al., 2005), pela ausência de padronização das doses aplicadas nos experimentos e pelas diferenças nas características dos solos (Schomberg et al., 2009).

A TMN média das duas áreas experimentais (28\%) foi muito próxima (Figura 3 ) do valor de $30 \%$ citado na Resolução no 375 (Brasil, 2006), para diferentes tipos de lodo. No entanto, os resultados obtidos no presente trabalho são indicativos de que a determinação da TMN em laboratório, específica para o solo em que se pretende aplicar o resíduo, é preferível a simples adoção de um valor predefinido em função do tipo de lodo.

Cabe destacar que os solos tratados com sucessivas aplicações de lodo aumentam sua capacidade de fornecer $\mathrm{N}$ para as plantas, como observado no presente trabalho e em outros (López-Tercero et al., 2005; Pitombo, 2011; Andrade et al., 2013). Esse aspecto, contudo, é negligenciado na recomendação da dose de lodo (Brasil, 2006). Portanto, deve-se aprimorar o sistema de recomendação da dose de lodo de esgoto e 
evitar a falta ou o excesso de $\mathrm{N}$, de forma a maximizar a eficiência de uso do nutriente. Nesse sentido, torna-se importante conhecer o aporte total de $\mathrm{N}$ disponibilizado a partir de novas doses de lodo adicionadas, além do efeito residual de aplicações anteriores.

A correlação entre o $\mathrm{N}$ mineralizado, determinado com o método anaeróbico, e o $\mathrm{N}$ absorvido pelo milho cultivado no campo foi estimada com uso dos dados de laboratório exatamente correspondentes às combinações de campo que avaliaram o efeito residual e o de novas aplicações, em cada área experimental (Figura 4). No experimento do IAC, o nitrogênio mineralizado correlacionou-se positivamente com a quantidade de nitrogênio absorvida pelo milho. $\mathrm{Na}$ área experimental da Embrapa Meio Ambiente, essa correlação foi maior do que a observada no IAC.

A avaliação da mineralização do $\mathrm{N}$ pelo método de incubação anaeróbica tem sido feita em diversas pesquisas, como na de Acosta (2009), que analisou amostras de solo com doses de resíduo vegetais, ou de Rhoden et al. (2006), que estudaram a mineralização
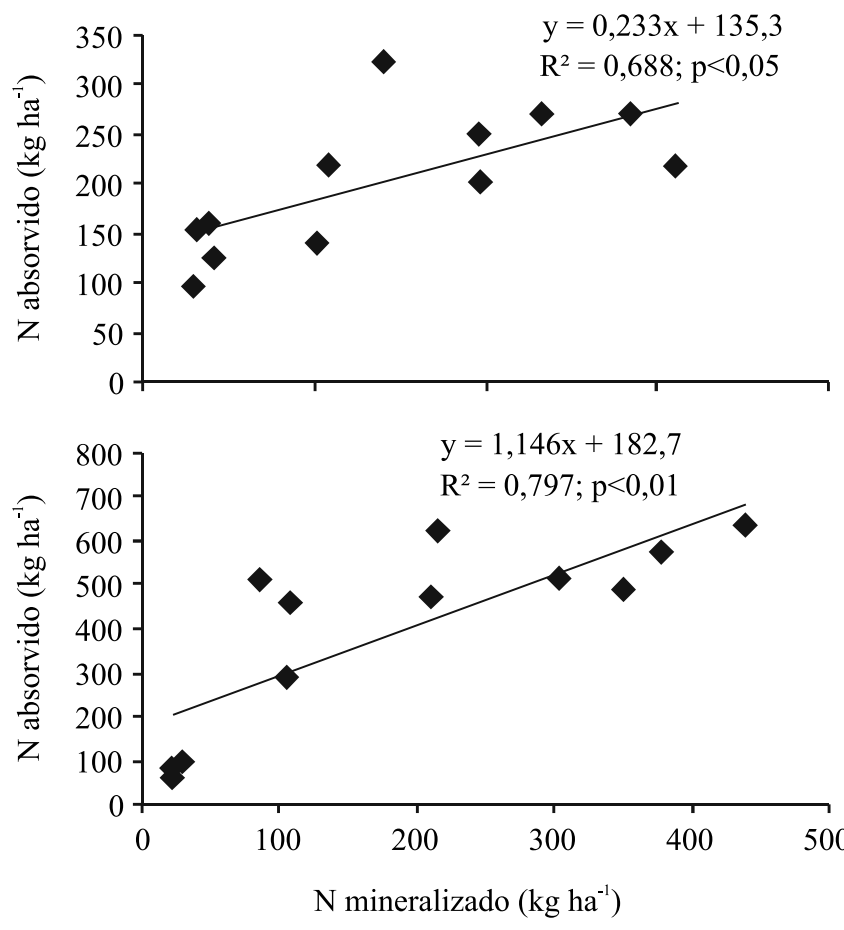

Figura 4. Regressão linear entre a quantidade de $\mathrm{N}$ mineralizado no solo e absorvido pelo milho: $\mathrm{A}$, área experimental do Instituto Agronômico; B, área experimental da Embrapa Meio Ambiente. do nitrogênio em 15 solos do Rio Grande do Sul. Em ambos os trabalhos, foram encontradas correlações positivas entre o $\mathrm{N}$ mineralizado, estimado com o método anaeróbico, e o $\mathrm{N}$ absorvido pelas plantas, com coeficientes de correlação de 0,90 e 0,70, respectivamente. Esses resultados, bem como os obtidos no presente trabalho, são indicativos de que o método de incubação anaeróbica é eficiente em quantificar a mineralização do $\mathrm{N}$.

\section{Conclusões}

1. A fertilização com lodo de esgoto é mais eficiente em fornecer nitrogênio, em longo prazo, do que a adubação mineral.

2. O efeito residual do lodo de esgoto, em solos tratados sucessivamente com o resíduo, não influencia a taxa de mineralização do nitrogênio proveniente da dose de resíduo recém-adicionado ao solo.

3. O método de incubação anaeróbica é eficiente em estimar a mineralização de nitrogênio de doses de lodo de esgoto recém-adicionadas ao solo.

\section{Agradecimentos}

À Coordenação de Aperfeiçoamento de Pessoal de Nível Superior (Capes), pela concessão de bolsa; e ao Conselho Nacional de Desenvolvimento Científico e Tecnológico $(\mathrm{CNPq})$, pelo apoio financeiro.

\section{Referências}

ACOSTA, J.A.A. Dinâmica do nitrogênio sob sistema plantio direto e parâmetros para o manejo da adubação nitrogenada no milho. 2009. 200p. Tese (Doutorado) - Universidade Federal de Santa Maria, Santa Maria.

ADAMS, M.A.; ATTIWILL, P.M. Nutrient cycling and nitrogen mineralization in eucalypt forests of south-eastern Australia. II. Indices of nitrogen mineralization. Plant and Soil, v.92, p.341-362, 1986. DOI: $10.1007 / \mathrm{BF} 02372482$.

ALCÂNTARA, M.A.K. de; AQUINO NETO, V. de; CAMARGO, O.A. de; CANTARELLA, H. Mineralização do nitrogênio em solos tratados com lodos de curtume. Pesquisa Agropecuária Brasileira, v.42, p.547-555, 2007. DOI: 10.1590/S0100-204X2007000400013.

ANDRADE, C.A. de; BOEIRA, R.C.; PIRES, A.M.M. Nitrogênio presente em lodo de esgoto e a resolução no 375 do Conama. In: COSCIONE, A.R.; NOGUEIRA, T.A.R.; PIRES, A.M.M. (Ed.). Uso agrícola de lodo de esgoto: avaliação após resolução $\mathrm{n}^{\circ} 375$ do Conama. Botucatu: FEPAF, 2010. p.157-170.

ANDRADE, C.A. de; SILVA, L.F.M.; PIRES, A.M.M.; COSCIONE, A.R. Mineralização do carbono e do nitrogênio 
no solo após sucessivas aplicações de lodo de esgoto. Pesquisa Agropecuária Brasileira, v.48, p.536-544, 2013. DOI: 10.1590/ S0100-204X2013000500010.

BARBOSA, G.M. de C.; TAVARES FILHO, J.; BRITO, O.B.; FONSECA, I.C.B. Efeito residual do lodo de esgoto na produtividade do milho safrinha. Revista Brasileira de Ciência do Solo, v.31, p.601-605, 2007. DOI: 10.1590/S0100-06832007000300020.

BETTIOL, W.; CAMARGO, O.A. de; GALVÃO, J.A.H.; GHINI, R. Impacto ambiental do uso agrícola do lodo de esgoto: descrição do estudo. In: BETTIOL, W.; CAMARGO, O.A. de (Ed.). Lodo de esgoto: impactos ambientais na agricultura. Jaguariúna: Embrapa Meio Ambiente, 2006. p.17-23.

BOEIRA, R.C. Aspectos experimentais na avaliação da mineralização de nitrogênio de lodos de esgoto incubados com solos. Jaguariúna: Embrapa Meio Ambiente, 2005. 5p. (Embrapa Meio Ambiente. Comunicado técnico, 27).

BOEIRA, R.C.; LIGO, M.A.V.; DYNIA, J.F. Mineralização de nitrogênio em solo tropical tratado com lodos de esgoto. Pesquisa Agropecuária Brasileira, v.37, p.1639-1647, 2002. DOI: 10.1590/ S0100-204X2002001100016

BOEIRA, R.C.; MAXIMILIANO, V.C.B. Mineralização de compostos nitrogenados de lodos de esgoto na quinta aplicação em Latossolo. Revista Brasileira de Ciência do Solo, v.33, p.711-722, 2009. DOI: 10.1590/S0100-06832009000100022.

BRASIL. Ministério do Meio Ambiente. Conselho Nacional do Meio Ambiente. Resolução no 375, de 29 de agosto de 2006. Define critérios e procedimentos, para o uso agrícola de lodos de esgoto gerados em estações de tratamento de esgoto sanitário e seus produtos derivados, e dá outras providências. Diário Oficial [da] República Federativa do Brasil, 30 ago. 2006.

BUENO, J.R.P.; BERTON, R.S.; SILVEIRA, A.P.D. da; CHIBA, M.K.; ANDRADE, C.A. de; DE MARIA, I.C. Chemical and microbiological attributes of an oxisol treated with successive applications of sewage sludge. Revista Brasileira de Ciência do Solo, v.35, p.1461-1470, 2011. DOI: 10.1590/ S0100-06832011000400040.

CABRERA, M.L.; KISSEL, D.E.; VIGIL, M.F. Nitrogen mineralization from organic residues: research opportunities. Journal of environmental quality, v.34, p.75-79, 2005. DOI: $10.2134 /$ jeq2005.0075.

CANTARELLA, H.; ANDRADE, C.A.; MATTOS JUNIOR, D. de. Matéria orgânica do solo e disponibilidade de $\mathrm{N}$ para as culturas. In: SANTOS, G. de A.; SILVA, L.S. da; CANELLAS, L.P.; CAMARGO, F.A.O. (Ed.). Fundamentos da matéria orgânica do solo: ecossistemas tropicais e subtropicais. Porto Alegre: Metrópole, 2008. p.581-596.

CANTARELLA, H.; DUARTE, A.P. Manejo da fertilidade do solo para a cultura do milho. In: GALVÃO, J.C.C.; MIRANDA, G.V. (Ed.). Tecnologia de produção de milho. Viçosa: Ed. da UFV, 2004. p.139-182.

CORREAA, R.S.; WHITE, R.E.; WEATHERLEY, A.J. Effects of sewage sludge stabilization on organic-N mineralization in two soils. Soil Use and Management, v.28, p.12-18, 2012. DOI: 10.1111/j.1475-2743.2012.00387.x.
DYNIA, J.F.; SOUZA, M.D. de; BOEIRA, R.C. Lixiviação de nitrato em Latossolo cultivado com milho após aplicações sucessivas de lodo de esgoto. Pesquisa Agropecuária Brasileira, v.41, p.855-862, 2006. DOI: 10.1590/ S0100-204X2006000500019.

GONÇALVES, F.T. de A. Dinâmica do nitrogênio em solo tratado com lodo de esgoto e cultivado com café. 2005. 73p. Dissertação (Mestrado) - Instituto Agronômico de Campinas, Campinas.

KAMPHAKE, L.J.; HANNAH, S.A.; COEHN, J.M. Automated analysis for nitrate by hydrazine reduction. Water Research, v.1, p.205-216, 1967. DOI: 10.1016/0043-1354(67)90011-5.

KEENEY, D.R. Nitrogen-availability indices. In: PAGE, A.L. (Ed.). Methods of soil analysis. Part 2. Chemical and microbiological properties. $2^{\text {nd }}$ ed. Madison: American Society of Agronomy: Soil Science Society of America, 1982. p.711-733.

KROM, M.D. Spectrophotometric determination of ammonia: a study of a modified Berthelot reaction using salicylate and dichloroisocyanurate. Analyst, v.105, p.305-316, 1980. DOI: 10.1039/an9800500305.

KUZYAKOV, Y. Priming effects: interactions between living and dead organic matter. Soil Biology and Biochemistry, v.42, p.1363-1371, 2010. DOI: 10.1016/j.soilbio.2010.04.003.

LÓPEZ-TERCERO, A.M; ANDRADE, M.L.; MARCET, P. Organic nitrogen mineralization rate in sewage sludge-amended mine soil. Communications in Soil Science and Plant Analysis, v.36, p.1005-1019, 2005. DOI: 10.1081/CSS-200050410.

PITOMBO, L.M. Estoques de carbono e nitrogênio e fluxos de gases do efeito estufa em solo com diferentes históricos de aplicação de lodo de esgoto. 2011. 61p. Dissertação (Mestrado) Instituto Agronômico, Campinas.

RHODEN, A.C.; SILVA, L.S. da; CAMARGO, F.A. de O.; BRITZKE, D.; BENEDETTI, E.L. Mineralização anaeróbia do nitrogênio em solos de várzea do Rio Grande do Sul. Ciência Rural, v.36, p.1780-1787, 2006. DOI: 10.1590/ S0103-84782006000600017.

SANTOS, H.G. dos; JACOMINE, P.K.T.; ANJOS, L.H.C. dos; OLIVEIRA, V.A. de; OLIVEIRA, J.B. de; COELHO, M.R.; LUMBRERAS, J.F.; CUNHA, T.J.F. (Ed.). Sistema brasileiro de classificação de solos. 2.ed. Rio de Janeiro: Embrapa Solos, 2006. $306 \mathrm{p}$.

SCHOMBERG, H.H.; WIETHOLTER, S.; GRIFFIN, T.S.; REEVES, D.W.; CABRERA, M.L.; FISHER, D.S.; ENDALE, D.M.; NOVAK, J.M.; BALKCOM, K.S.; RAPER, R.L.; KITCHEN, N.R.; LOCKE, M.A.; POTTER, K.N.; SCHWARTZ, R.C.; TRUMAN, C.C.; TYLER, D.D. Assessing indices for predicting potential nitrogen mineralization in soils under different management systems. Soil Science Society of America Journal, v.73, p.1575-1586, 2009. DOI: 10.2136/ sssaj2008.0303.

STEVENSON, F.J. Cycles of soil: carbon, nitrogen, phosphorus, sulfur, micronutrients. New York: J. Wiley, 1986. 380p.

TAIZ, L.; ZEIGER, E. Plant physiology. $2^{\text {nd }}$ ed. Sunderland: Sinauer, 1998. 792p.

Pesq. agropec. bras., Brasília, v.50, n.4, p.333-342, abr. 2015 DOI: 10.1590/S0100-204X2015000400009 
VAN RAIJ, B.; CANTARELLA, H.; QUAGGIO, J.A.; FURLANI, A.M.C. (Ed.) Recomendações de adubação e calagem para o Estado de São Paulo. 2.ed. Campinas: Instituto Agronômico, 1997. 285p. (IAC. Boletim técnico, 100).

VIEIRA, R.F.; CARDOSO, A.A. Variações nos teores de nitrogênio mineral em solo suplementado com lodo de esgoto. Pesquisa Agropecuária Brasileira, v.38, p.867-874, 2003. DOI: 10.1590/ S0100-204X2003000700011.
WIENHOLD, B.J. Comparison of laboratory methods and an in situ method for estimating nitrogen mineralization in an irrigated siltloam soil. Communications in Soil Science and Plant Analysis, v.38, p.1721-1732, 2007. DOI: 10.1080/00103620701435498.

YAGI, R.; FERREIRA, M.E.; CRUZ, M.C.P. da; BARBOSA, J.C. Mineralização potencial e líquida de nitrogênio em solos. Revista Brasileira de Ciência do Solo, v.33, p.385-394, 2009. DOI: 10.1590/S0100-06832009000200016.

Recebido em 21 de agosto de 2014 e aprovado em 2 de março de 2015 